\title{
Development of radar cross section analysis system of naval ships
}

\author{
Kookhyun $\mathrm{Kim}^{1}$, Jin-Hyeong $\mathrm{Kim}^{2}$, Tae-Muk $\mathrm{Choi}^{2}$ and Dae-Seung Cho ${ }^{3}$ \\ ${ }^{1}$ Dept. of Naval Architecture, Tongmyong University, Busan, Korea \\ ${ }^{2}$ Createch Co., Ltd., Busan, Korea \\ ${ }^{3}$ Dept. of Naval Architecture and Ocean Engineering, Pusan National University, Busan, Korea
}

\begin{abstract}
A software system for a complex object scattering analysis, named SYSCOS, has been developed for a systematic radar cross section (RCS) analysis and reduction design. The system is based on the high frequency analysis methods of physical optics, geometrical optics, and physical theory of diffraction, which are suitable for RCS analysis of electromagnetically large and complex targets as like naval ships. In addition, a direct scattering center analysis function has been included, which gives relatively simple and intuitive way to discriminate problem areas in design stage when comparing with conventional image-based approaches. In this paper, the theoretical background and the organization of the SYSCOS system are presented. To verify its accuracy and to demonstrate its applicability, numerical analyses for a square plate, a sphere and a cylinder, a weapon system and a virtual naval ship have been carried out, of which results have been compared with analytic solutions and those obtained by the other existing software.
\end{abstract}

KEY WORDS: Radar cross section analysis; Naval ship; Combining method of physical optics and geometric optics; Physical theory of diffraction; Direct scattering center analysis; Graphical user interface.

\section{INTRODUCTION}

The radar cross section (RCS) reduction of a naval ship is one of the most important design issues when considering the survivability in a hostile environment. Therefore, in design phase, the RCS reduction techniques such as shaping, shielding and applying radar absorbing material are considered and their effectiveness are examined by the RCS assessment including the discrimination of problem areas by the scattering center analysis.

For this purpose, various codes have been developed by some institutes and applied to military industries. For examples, there are SIGNET/RCS (BAE SYSTEMS, 2004), EPSILON (Simpson, et al., 1998), RAMSES (IABG, 2002), XPATCH (Lee, et al., 1993) and CADRCS (Biegel, et al., 2004). Most of the codes have adopted the high frequency methods such as physical optics (PO), geometric optics (GO), physical theory of diffraction (PTD), geometrical theory of diffraction (GTD) which are suitable for the RCS analysis of large and complex targets such as naval ships. For the military security reason, however, the institutes generally hesitate to open the actual code to other countries.

In the meantime, some studies on RCS analysis techniques have been carried out in Korea. Suk, et al. (2001) have proposed a multi-resolution grid algorithm to alleviate the computational cost of the conventional shooting and bouncing ray method (SBR) proposed by Lee, et al. (1988). Kim, et al. (2005a) have proposed an object precision method (OPM) for RCS analysis of the complex target consisting of perfect electric conductive (PEC) patches. Kim, et al. (2009) have also suggested a combining method of PO and GO to consider the effect of non-PEC surfaces such as ocean surface, which has been derived from Stratton-Chu integral equation by applying a Kirchhoff approximation and by assuming a far field condition. Kim, et al. (2007a) have presented a numerical scheme to calculate the edge diffraction of rotating targets based on PTD. Kim, et al. (2005b) have proposed a direct scattering center analysis method based on the OPM, instead of conventional image-based

Corresponding author: Dae-Seung Cho

e-mail: daecho@pusan.ac.kr 
approaches such as range-profile and synthetic aperture radar. The method is able to simply and intuitively discriminate the problem areas from the colored RCS levels on the numerical model. Despite of these studies, additional effort is necessary to develop a system for efficient and systematic RCS analysis and reduc-tion design of large and complex targets.

In this study, a software system for RCS analysis of electromagnetically large and complex targets, named SYSCOS, has been developed. The system is theoretically based on the high-frequency analysis methods of PO, GO, and PTD. The system also contains a function for efficient discrimination of problem areas based on the direct scattering center analysis method. In this paper, the theoretical background and the organization of the presented system are introduced. To verify its accuracy and to demonstrate its applicability, numerical analyses for a square plate, a sphere and a cylinder, a weapon system and a virtual naval ship have been carried out, of which results have been compared with analytic solutions and those obtained by the existing software.

\section{THEORETICAL BACKGROUND}

\section{RCS matrix}

The RCS measure considering a polarization of electromagnetic waves in spherical coordinates is defined as the following 2 $\times 2$ RCS matrix $[\sigma]$ (Knott, et al., 1993).

$$
[\sigma]=\left[\begin{array}{ll}
\sigma_{\theta \theta} & \sigma_{\theta \phi} \\
\sigma_{\phi \theta} & \sigma_{\phi \phi}
\end{array}\right]
$$

where $\sigma_{u v}$ is the $u v$-polarization component of the RCS matrix $(u, v=\theta, \phi) . \theta$ and $\phi$ are the azimuth angle and the eleva-tion angle, respectively.

In the meantime, the $u v$-polarized RCS of a target in far-field condition is defined as the following equation.

$$
\sigma_{u v}=\lim _{R \rightarrow \infty}\left|4 \pi R^{2} \frac{\vec{E}_{s, u}}{\vec{E}_{i, v}}\right|^{2}
$$

where $R$ is the distance between the receiver and the center of the target, $\vec{E}_{s, u}$ and $\vec{E}_{i, v}$ are the scattered and incident electric field vectors of electromagnetic waves for the target.

\section{Physical Optics (PO)}

Assuming the plane wave incidence to a target, the electric field vector scattered from the target surface to a receiver $\vec{E}_{s}$ is calculated with the following Stratton-Chu integral equation (Knott, et al., 1993).

$$
\vec{E}_{s}=-\frac{j k e^{-j k R}}{4 \pi R} \int_{s}\left\{\hat{\zeta}_{s} \times\left[\hat{n} \times \vec{E}-\eta \hat{\zeta}_{s} \times(\hat{n} \times \vec{H})\right]\right\} e^{j k \vec{r} \cdot\left(\hat{\zeta}_{s}-\hat{\zeta}_{i}\right)} d S
$$

where $j$ is the unit imaginary, $S$ is the target surface, $k$ is the wave number $(=\omega / c), \omega$ is the circular frequency, and $c$ is the speed of electromagnetic waves. $\hat{\zeta}_{s}$ and $\hat{\zeta}_{i}$ are the unit directional vectors of the incidence and scattering of the electromagnetic wave, respectively. $\hat{n}$ is the unit normal vector of the target surface. $\vec{E}$ and $\vec{H}$ are the electric field vector and the magnetic field vector induced on the surface, respectively. $\vec{r}$ is the position vector of a certain point on the target surface. $\eta$ is the elec-tromagnetic impedance of the medium (e.g. air).

Assume that the target surface is flat and the local coordinates is defined as shown in Fig. 1. By applying Kirchhoff approximation, the equation (3) can be represented as the following equation.

$$
\vec{E}_{s}=-\frac{j k e^{-j k R}}{2 \pi R} E_{0} \vec{W}(\hat{p}) \int_{s} e^{j k \vec{r} \cdot\left(\hat{\zeta}_{s}-\hat{\zeta}_{i}\right)} d S
$$

where $E_{o}\left(=\left|\vec{E}_{i}\right|\right)$ is the magnitude of the incident electric field vector $\vec{E}_{i} . \vec{W}(\hat{p})$ is the polarization vector with respect to the unit polarization vector $\hat{p}\left(=\vec{E}_{i} / E_{o}\right)$ and is represented as the following equation. 


$$
\begin{aligned}
\vec{W}(\hat{p})= & \frac{1}{2} \hat{\zeta}_{s} \times\left\{\left(1+\Gamma_{E}\right)\left(\hat{p} \cdot \hat{e}_{\perp}\right)\left(\hat{n} \times \hat{e}_{\perp}\right)+\left(1-\Gamma_{H}\right)\left(\hat{p} \cdot \hat{e}_{\|}^{i}\right)\left(\hat{\zeta}_{i} \cdot \hat{n}\right) \hat{e}_{\perp}\right. \\
& \left.+\left(1-\Gamma_{E}\right)\left(\hat{p} \cdot \hat{e}_{\perp}\right)\left(\hat{\zeta}_{i} \cdot \hat{n}\right)\left(\hat{\zeta}_{s} \times \hat{e}_{\perp}\right)-\left(1+\Gamma_{H}\right)\left(\hat{p} \cdot \hat{e}_{\|}^{i}\right)\left[\hat{\zeta}_{s} \times\left(\hat{n} \times \hat{e}_{\perp}\right)\right]\right\}
\end{aligned}
$$

where $\Gamma_{E}$ and $\Gamma_{H}$ are the Fresnel reflection coefficients for $E$ - and $H$-polarization, respectively. $\hat{e}_{\perp}$ and $\hat{e}_{\|}^{i}$ are the unit vertical and parallel vectors of the incident wave with respect to the incident plane, respectively.

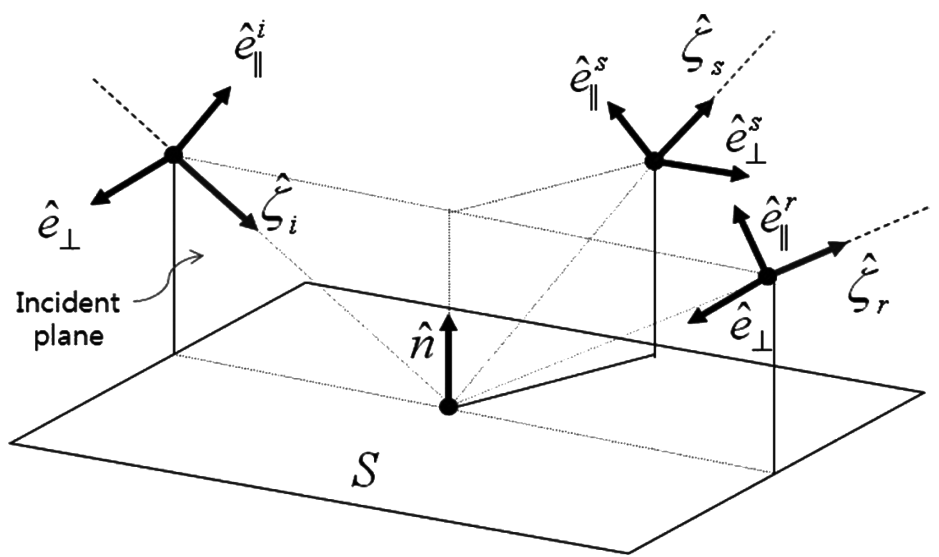

Fig. 1 Definition of local coordinates.

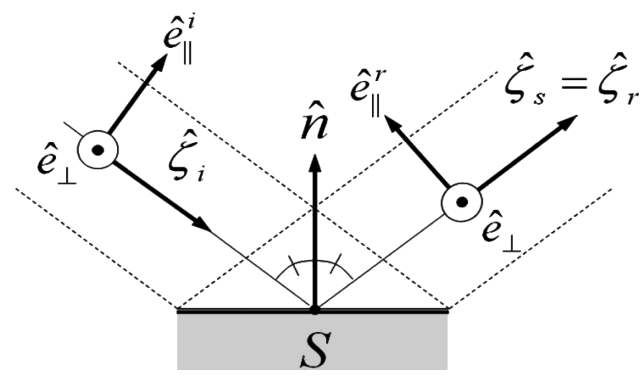

Fig. 2 Definition of local coordinates for specular reflection of electromagnetic wave.

\section{Geometric Optics (GO)}

In high-frequency range, it is assumed that the electromagnetic wave propagates straightly in a homogeneous medium and the incident wave is reflected toward the specular direction by Snell's law as shown in Fig. 2. The direction of the scattering $\hat{\zeta}_{s}$ is coincident with that of specular reflection $\hat{\zeta}_{r}$, and the polarization vector of the equation (5) can be simplified to the following equation.

$$
\vec{W}(\hat{p})=-\left[\Gamma_{E}\left(\hat{p} \cdot \hat{e}_{\perp}\right) \hat{e}_{\perp}+\Gamma_{H}\left(\hat{p} \cdot \hat{e}_{\|}^{i}\right) \hat{e}_{\|}^{r}\right]\left(\hat{\zeta}_{s} \cdot \hat{n}\right)
$$

where $\hat{e}_{\|}^{r}$ is the parallel unit vector of scattered wave with respect to the incident plane.

\section{Combining method of PO and GO}

PO and GO described above must be good approximate methods for RCS of electromagnetically large targets, when the single reflection effect is dominant and shadowing effects are negligible. There, however, exist limitations on applying to complex-shaped target as like naval ships whose multiple reflection and shadowing effects could not be negligible. Therefore a combining method of PO and GO applying GO for multiple reflections and PO for the last reflection (Kim, et al., 2009) is adopted in SYSCOS.

By introducing a local coordinate transformation matrix, the $u v$-polarized RCS of the target consisting of $N_{r}$ scatters can be represented as the following equation. 


$$
\sigma_{u v}=\frac{k^{2}}{\pi}\left|\sum_{n=1}^{N_{r}} p_{u v}^{n} \int_{S_{n}} e^{j k \Theta_{n}} d S\right|^{2}
$$

where $S_{n}$ is the virtual surface corresponding to the $n$th scatter which can be extracted by using the hidden surface removal algorithm and the virtual surface concept (Klement, et al., 1988). $P_{u v}^{n}$ is the $u v$-component of the polarization matrix for the $n$th scatter which can be calculated by the following equation.

$$
[P]^{n}=\prod_{m=1}^{M}\left\{[T]_{m}^{n}[P]_{m}^{n}\right\}
$$

where

$$
\begin{aligned}
& {[T]_{m}^{n}=\left\{\begin{array}{c}
{\left[\begin{array}{cc}
\hat{\theta}_{i} \cdot \hat{e}_{\|, 1}^{i, n} & -\hat{\phi}_{i} \cdot \hat{e}_{\|, 1}^{i, n} \\
\hat{\theta}_{i} \cdot \hat{e}_{\perp, 1}^{n} & -\hat{\phi}_{i} \cdot \hat{e}_{\perp, 1}^{n}
\end{array}\right] \quad, m=1} \\
{\left[\begin{array}{cc}
\hat{e}_{\|, m-1}^{r, n} \cdot \hat{e}_{\|, m}^{i, n} & \hat{e}_{\perp, m-1} \cdot \hat{e}_{\|, m}^{i, n} \\
\hat{e}_{\|, m-1}^{r, n} \cdot \hat{e}_{\perp, m}^{i, n} & \hat{e}_{\perp, m-1} \cdot \hat{e}_{\perp, m}^{i, n}
\end{array}\right], m \neq 1}
\end{array}\right.} \\
& {[P]_{m}^{n}=\left\{\begin{array}{c}
{\left[\begin{array}{cc}
\Gamma_{H}^{n} & 0 \\
0 & \Gamma_{E}^{n}
\end{array}\right] \quad, m \neq M} \\
{\left[\begin{array}{cc}
\hat{\theta}_{s} \cdot \vec{W}\left(\hat{e}_{\|, m}^{i, n}\right) & -\hat{\theta}_{s} \cdot \vec{W}\left(\hat{e}_{\perp, m}^{n}\right) \\
-\hat{\phi}_{s} \cdot \vec{W}\left(\hat{e}_{\|, m}^{i, n}\right) & -\hat{\phi}_{s} \cdot \vec{W}\left(\hat{e}_{\perp, m}^{n}\right)
\end{array}\right], m=M}
\end{array}\right.}
\end{aligned}
$$

In the equation (9) and the equation (10), $\hat{\theta}$ and $\hat{\phi}$ are the unit vectors of the azimuth angle and the elevation angle in the spherical coordinates, respectively. Subscripts $i$ and $s$ mean the incidence and scattering of electromagnetic waves, respectively. $m$ and $M$ are the reflection order and the maximum one corresponding to the $n$th scatter, respectively. In the meantime, $\Theta_{n}$ in equation (7) is the virtual phase of the $n$th scatter, which can be calculated by the following equation.

$$
\Theta_{n}=\left(\vec{r}_{0, M}^{n}+\vec{r}_{0,1}^{n}\right) \cdot \hat{\zeta}_{s}^{n}-\sum_{m=1}^{M}\left\{||_{0, m-1}^{n}-\vec{r}_{0, m}^{n} \mid+\vec{r} \cdot\left(\hat{\zeta}_{s}^{n}-\hat{\zeta}_{M-1}^{n}\right)\right\}
$$

where $\vec{r}_{0, m}^{n}$ and $\hat{\zeta}_{m}^{n}$ are the position vector and the unit directional vector of the $m$ th order reflection, respectively.

\section{Physical theory of diffraction}

Essentially, PO and GO are inaccurate for off-normal incidence of the electromagnetic waves to the target surface. To mitigate this drawback, a numerical method based on PTD has been adopted in SYSCOS.

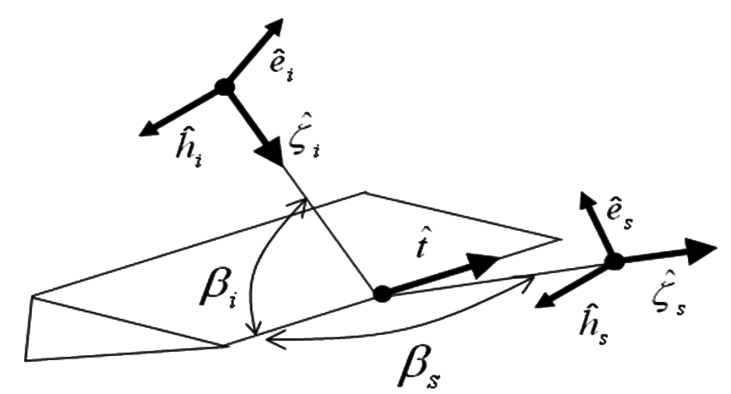

Fig. 3 Three dimensional diffraction by edge.

Assuming the incidence of the electromagnetic wave to a three dimensional edge as shown in Fig. 3, the electric vector field diffracted from the edge to a receiver can be calculated by the following equation (Knott, et al., 1993).

$$
\vec{E}_{s}=\frac{j k e^{-j k R}}{4 \pi R} \int_{t}\left\{\eta I_{e}\left[\hat{\zeta}_{s} \times\left(\hat{\zeta}_{s} \times \hat{t}\right)\right]+I_{m}\left(\hat{\zeta}_{s} \times \hat{t}\right)\right\} e^{j k\left(\vec{r}_{e} \cdot \vec{\zeta}_{s}\right)} d t
$$


where $t$ and $\hat{t}$ are the edge contour and the edge directional vector, respectively. $\vec{r}_{e}$ is the position vector of a certain point on the edge. $I_{e}$ and $I_{m}$ defined in Eq. (13) and Eq. (14) are the equivalent currents induced on the edge by the incident wave.

$$
\begin{aligned}
& I_{e}=\frac{2 j\left(\hat{t} \times \hat{e}_{i}\right) f}{\eta k \sin \beta_{i} \sin \beta_{s}} E_{o} e^{-j k\left(\vec{r}_{e} \cdot \hat{\zeta}_{i}\right)} \\
& I_{m}=\frac{2 j\left(\hat{t} \times \hat{h}_{i}\right) g}{k \sin \beta_{i} \sin \beta_{s}} E_{o} e^{-j k\left(\vec{r}_{e} \cdot \hat{\zeta}_{i}\right)}
\end{aligned}
$$

where $\hat{e}_{i}$ and $\hat{h}_{i}$ are unit vectors aligned along the incident electric and magnetic fields, respectively. $\beta_{i}$ and $\beta_{s}$ are the inner angles between the unit directional vectors of the incidence/scattering and the edge, respectively. Meanwhile, $f$ and $g$ are the Ufimtsev's diffraction coefficients which are similar with Keller's but modified by the PTD (Knott, et al., 1993).

When the edge of the target consists of the $N_{d}$ straight segments, by substituting the equation (12) to the equation (2) and considering monostatic case $\left(\hat{\zeta}_{s}=-\hat{\zeta}_{i}\right.$ and $\left.\beta_{i}=\beta_{s}\right)$, the $u v$-polarization components of RCS matrix in can be rewritten as

$$
\sigma_{u v}=\frac{k^{2}}{\pi}\left|\sum_{l=1}^{N_{d}}\left[\hat{u} \cdot \vec{W}_{d}^{l}(\hat{v})\right] e^{j k \bar{Z}_{l}} L_{l} \sin c\left(k L_{l} \cos \beta^{l}\right)\right|^{2}
$$

where $L_{l}$ and $\bar{Z}_{l}$ are the edge length and the distance from the edge center to the receiver, respectively. $\cos \beta^{l}$ is the absolute value of $\hat{\zeta}_{s} \times \hat{t}_{l} . l$ is the index for each segmented edge. $\vec{W}_{d}^{l}(\hat{v})$ defined in equation (16) is the polarization vector against the segmented edge.

$$
\vec{W}_{d}^{l}(\hat{v})=-\frac{j}{k \sin ^{2} \beta^{l}}\left\{\left(\hat{t}_{l} \cdot \hat{v}\right)\left[\hat{\zeta}_{s} \times\left(\hat{\zeta}_{s} \times \hat{t}_{l}\right)\right] f+\left[\hat{t}_{l} \cdot\left(\hat{\zeta}_{s} \times \hat{v}\right)\right]\left(\hat{\zeta}_{s} \times \hat{t}_{l}\right)\right\} g
$$

\section{Direct scattering center analysis method}

Conventional image-based approaches such as range-profile and synthetic aperture radar requiring another data process to discriminate scattering centers are ineffective in a practical point of view. Therefore, a direct method based on an OPM (Kim, et al., 2005a) has been adopted. The method directly displays RCS levels on the model surface with different colors, which enables to identify the scattering center intuitively (Kim, et al., 2005b). Fig. 4 shows the schematic concept of the RCS level coloring procedure for the case of multiple reflections. A wave field firstly induced on surface " $\mathrm{A}$ " impinges onto subsurface " $\mathrm{A}_{1}$ " of surface " $\mathrm{B}$ " and successively onto subsurface " $\mathrm{A}_{11}$ " of surface "C". Then, the RCS value of each subsurface due to single and multiple reflections are summed and displayed with a color considering its magnitude.

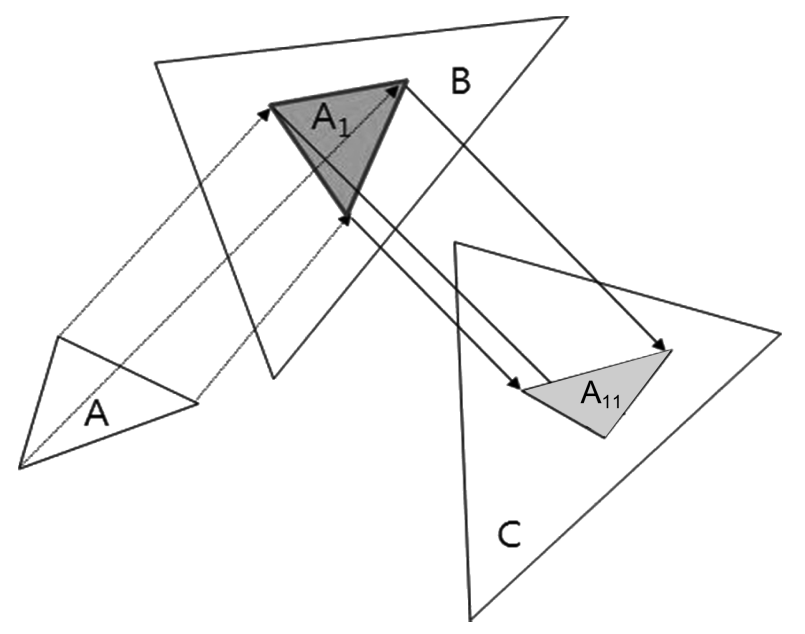

Fig. 4 RCS level-coloring procedure by direct scattering center analysis. 


\section{ORGANIZATION OF SYSCOS}

SYSCOS is mainly composed of three parts; preprocessor, main solver, and postprocessor as shown in Fig. 5.

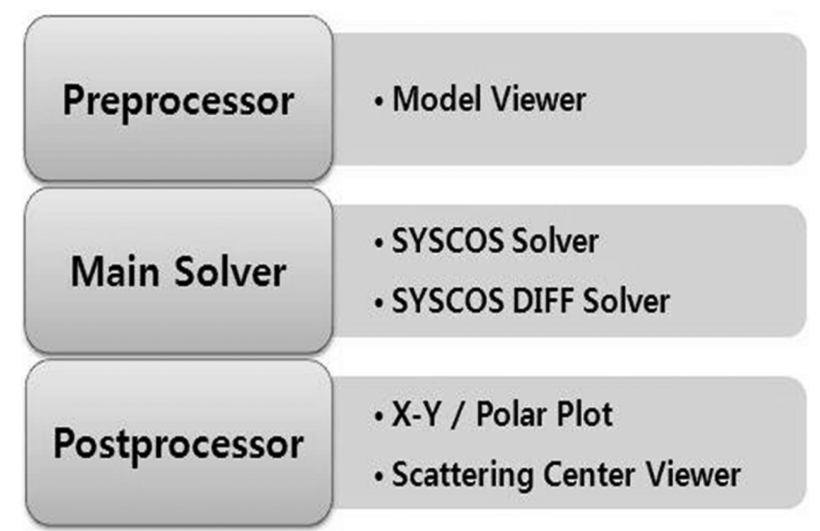

Fig. 5 Organization of SYSCOS.

\section{Preprocessor}

The model viewer shown in Fig. 6 provides functions to display numerical models, and to check the normal vectors of element surfaces. Particularly, the interface module gives a function to import the model data files from the generalized CAD software such as AUTOCAD (*.dxf) and MSC/ PATRAN (*.bdf).

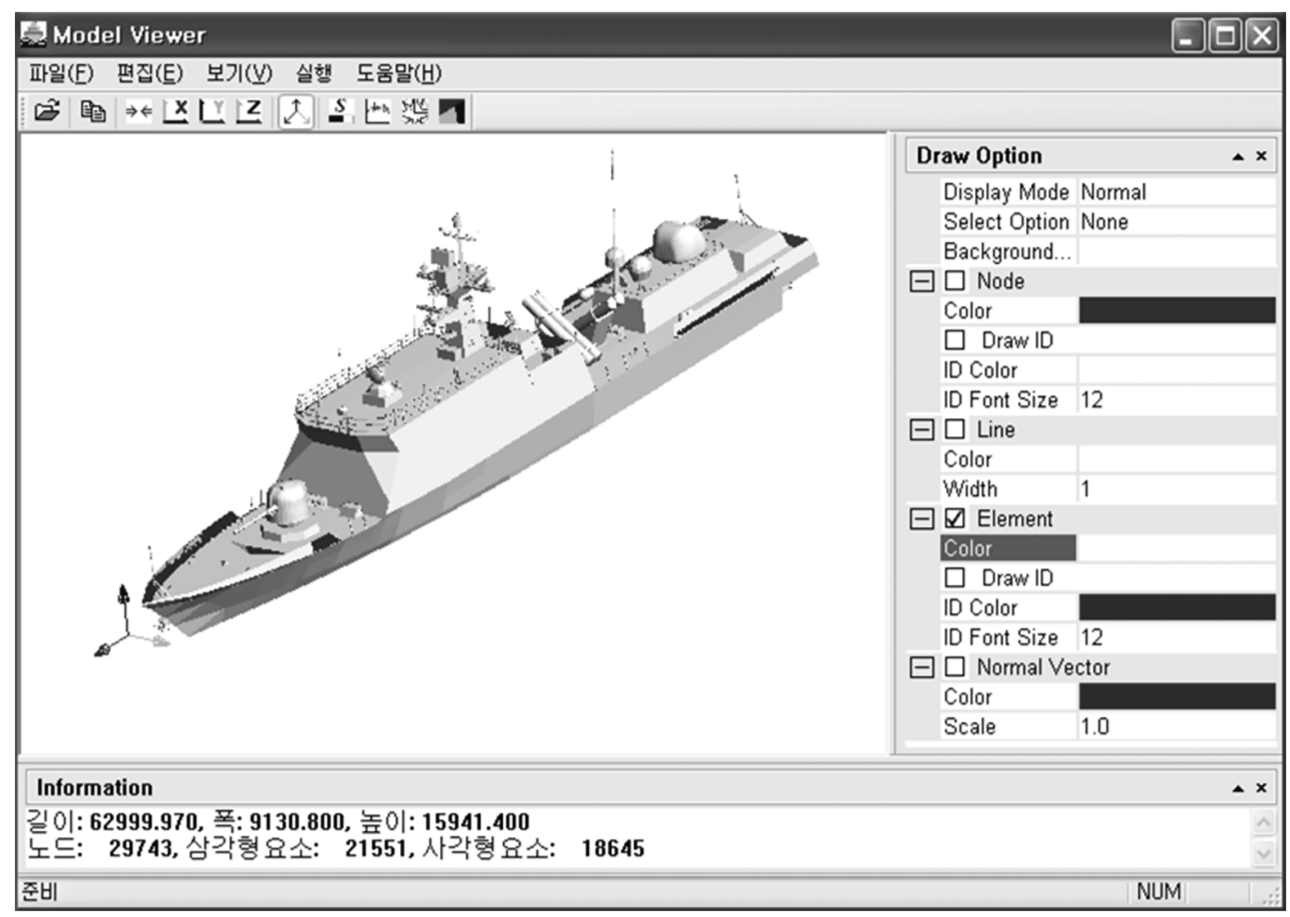

Fig. 6 Model viewer window.

\section{Main solver}

The main solver consists of SYSCOS solver and SYSCOS DIFF solver as shown in Fig. 7. The former is used for the RCS analysis only considering the specular reflections on the target surface, while the latter for the RCS analysis only considering the diffraction of the target edge. The objective target model and calculation options such as radar frequency, range of incident angle (azimuth and elevation), maximum number of reflection, and so on can be assigned. 
The output file contains analysis conditions, RCS values with respect to the incident angle, the polarizations, and the re-flection order as shown in Fig. 8.

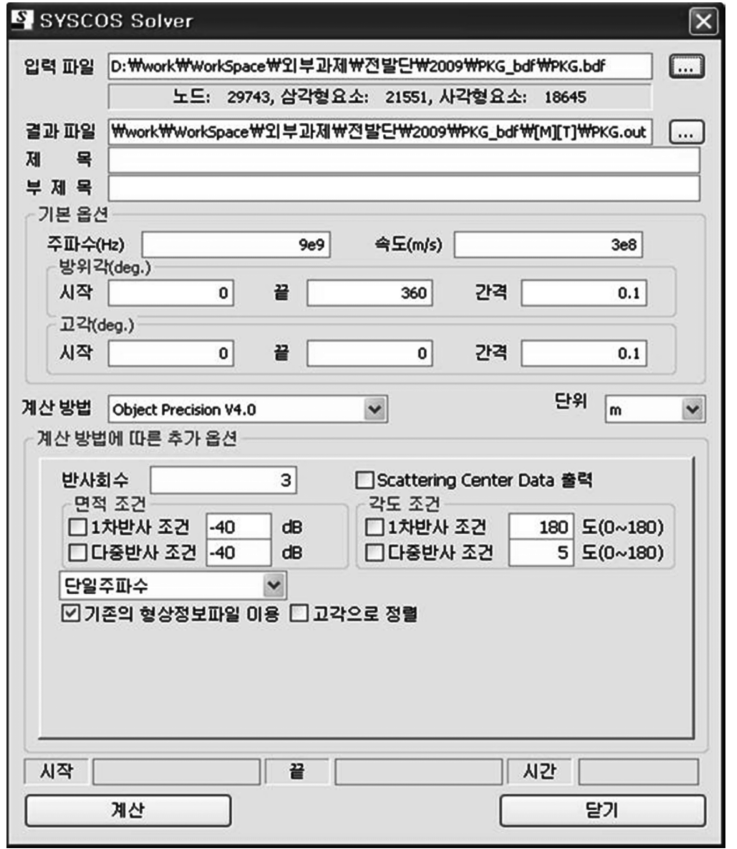

(a)

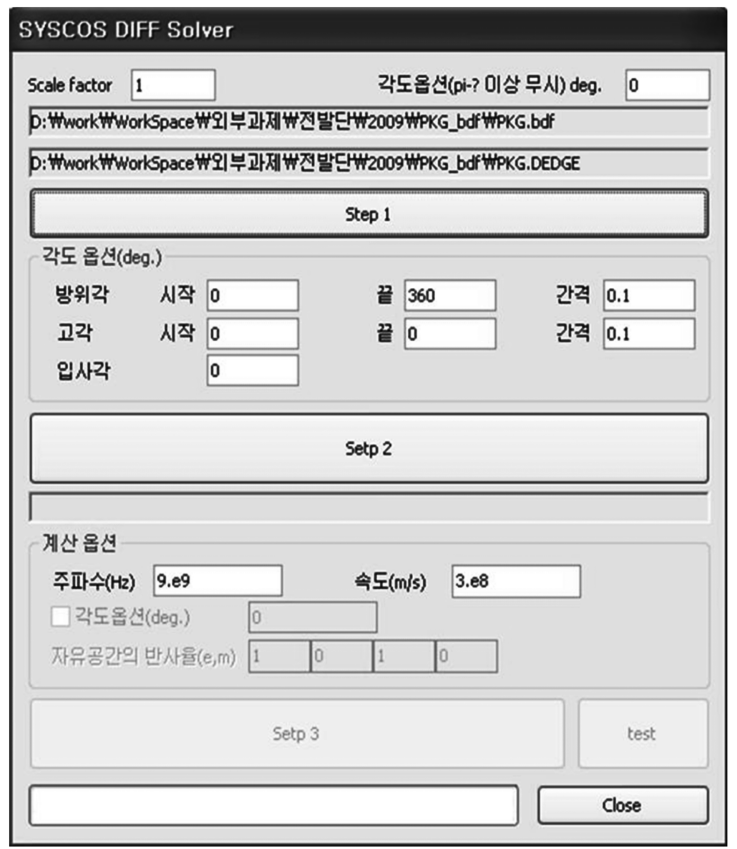

(b)

Fig. 7 Window for (a) SYSCOS solver and (b) SYSCOS DIFF solver.

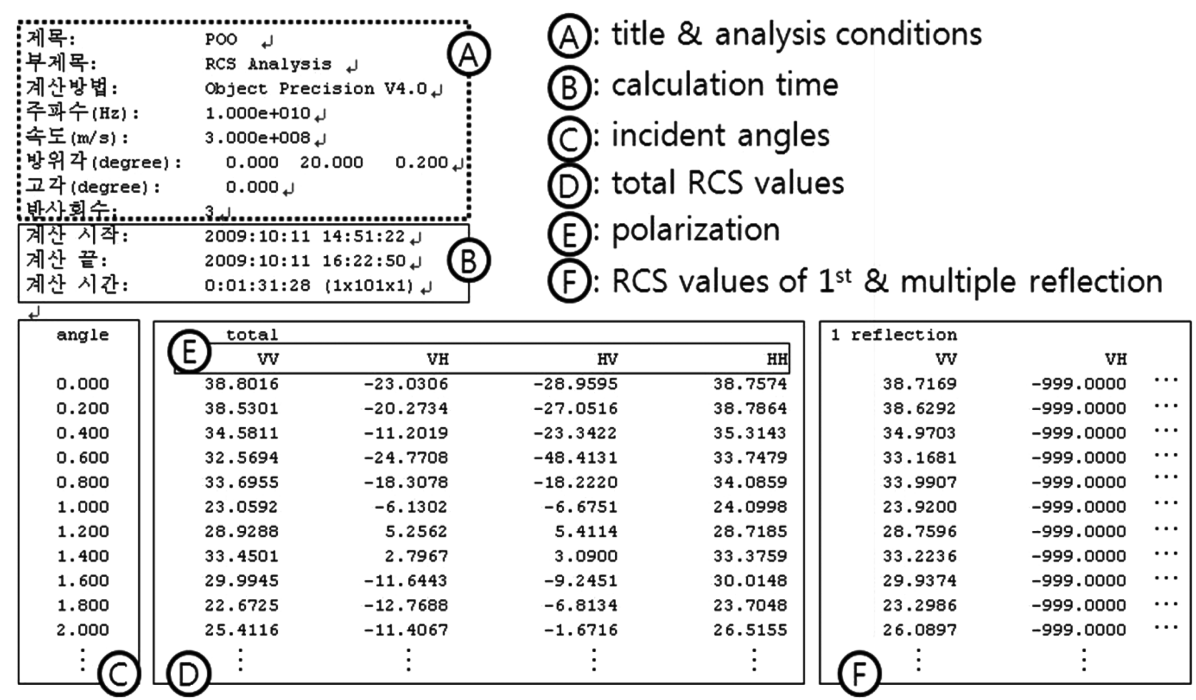

Fig. 8 Data structure of SYSCOS output file.

\section{Postprocessor}

The postprocessor consists of an X-Y plot, a polar plot and a scattering center viewer. The polar plot, shown in Fig. 9(a), is commonly used for representing the RCS analysis results in polar coordinates and for recognizing the angle of high RCS-valued peaks. The X-Y plot, shown in Fig. 9(b), is profitable to grasp the RCS level of the specified angle and provides a function of extracting statistical values such as maximum, minimum, mean, and median RCS values of the result. In addition, a window filter of raw data with respect to the azimuth angle is available in the X-Y plot and the window-filtered data is exporttable to the ASCII file. 


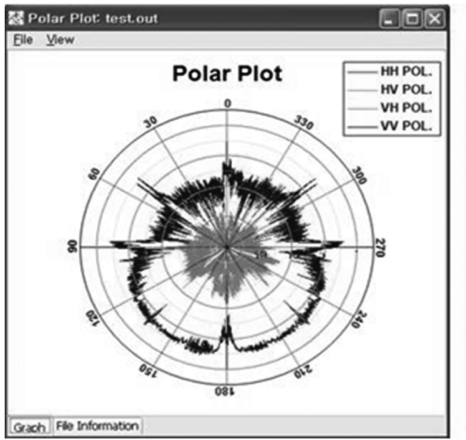

(a)

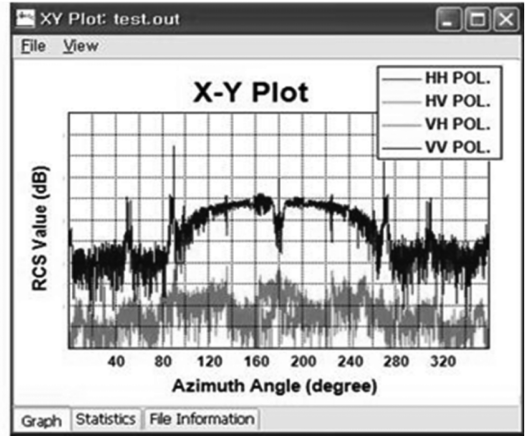

(b)

Fig. 9 Windows of (a) polar plot and (b) X-Y plot.

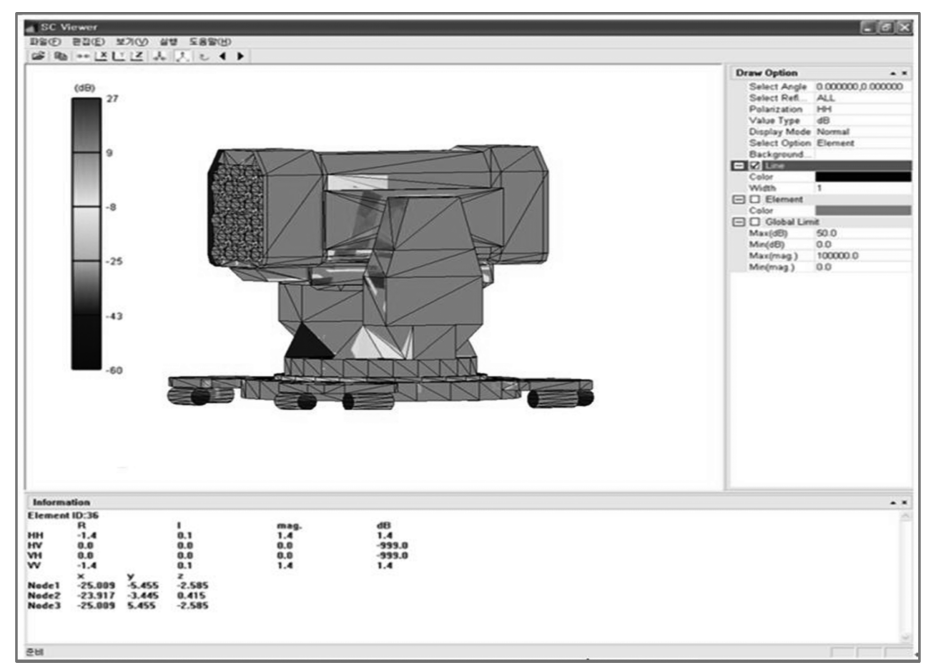

Fig. 10 Scattering center viewer window.

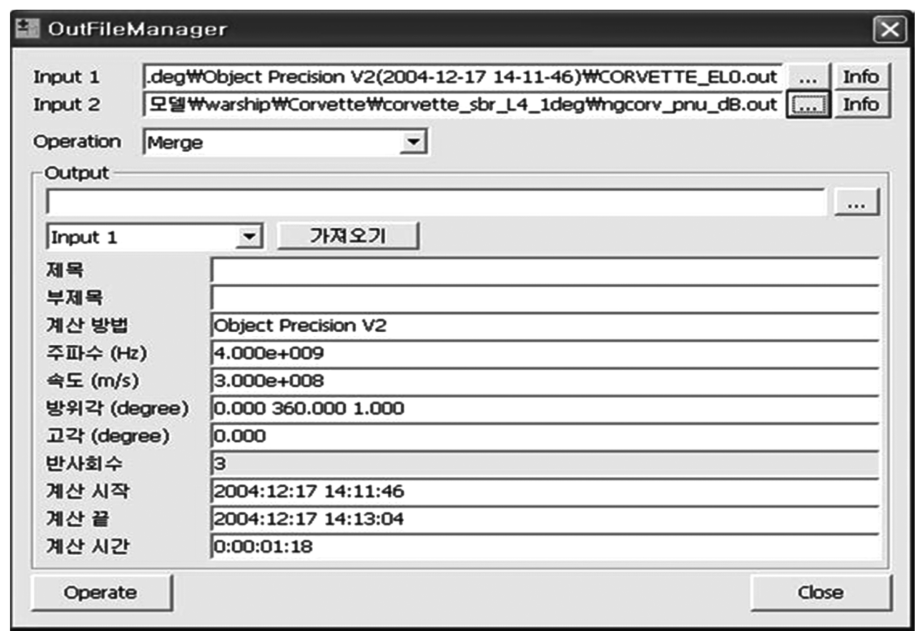

Fig. 11 Output files manager.

Also, the scattering center viewer, as shown in Fig. 10 as a sample, displays the RCS levels on numerical model using colors to easily identify scattering centers of the target. In the meantime, an output files manager shown in Fig. 11 provides a function for averaging, merging, summing, subtracting of the RCS analysis results. This function, in par-ticular, can raise the efficiency of the work when an engineer has to process massive data that are carried out on several PCs to reduce total calculation time. 


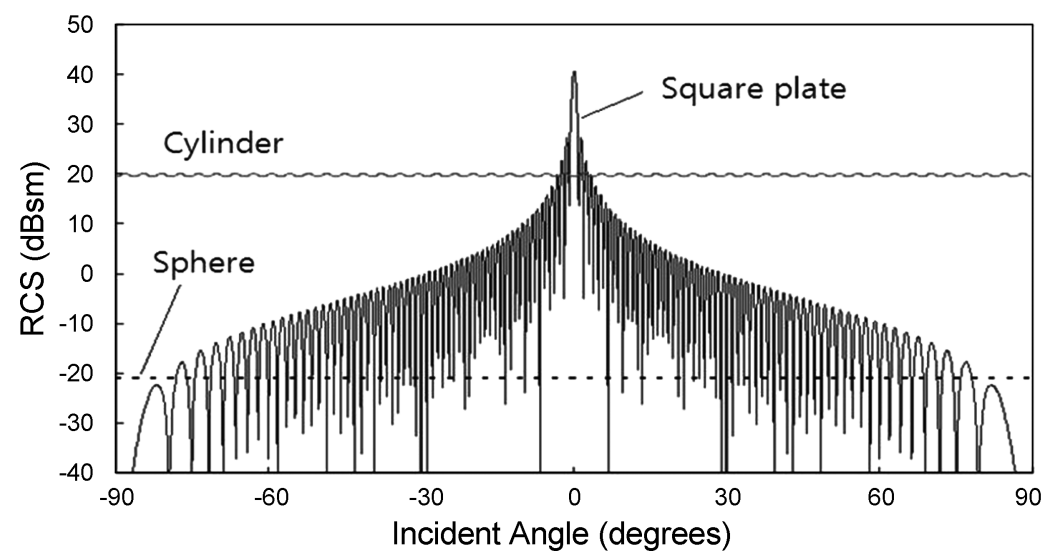

Fig. 12 RCS of the square plate, cylinder, and sphere.

\section{VALIDAIONS}

In order to verify the accuracy and to demonstrate applicability of the presented system, the RCS analyses have been carried out for simple and complex targets.

\section{Simple targets}

RCS analyses have been carried out for the simple PEC targets such as square plate, cylinder, sphere, dihedral, and two types of trihedrals, and it has been identified that the results calculated by the presented system have been well coincident with the theoretical results (Knott, et al., 1993) in RCS values and patterns. Among the results, the RCS values of the square plate, cylinder, and sphere are representatively shown in Fig. 12. In the calculation, the radar frequency is set to $9 \mathrm{GHz}$ and the reference size of each target such as height and width (or diameter) is set to $1 \mathrm{~m}$.

In the meantime, Fig. 13 shows the RCS analysis results of a rectangular plate with a size of $0.406 m \times 0.390 m$, where the frequency is set to $6.4 \mathrm{GHz}$. The result shows that the edge diffraction effect grows up, as the incident angle of the electromagnetic wave to the normal direction of the plate increases, as well-known.

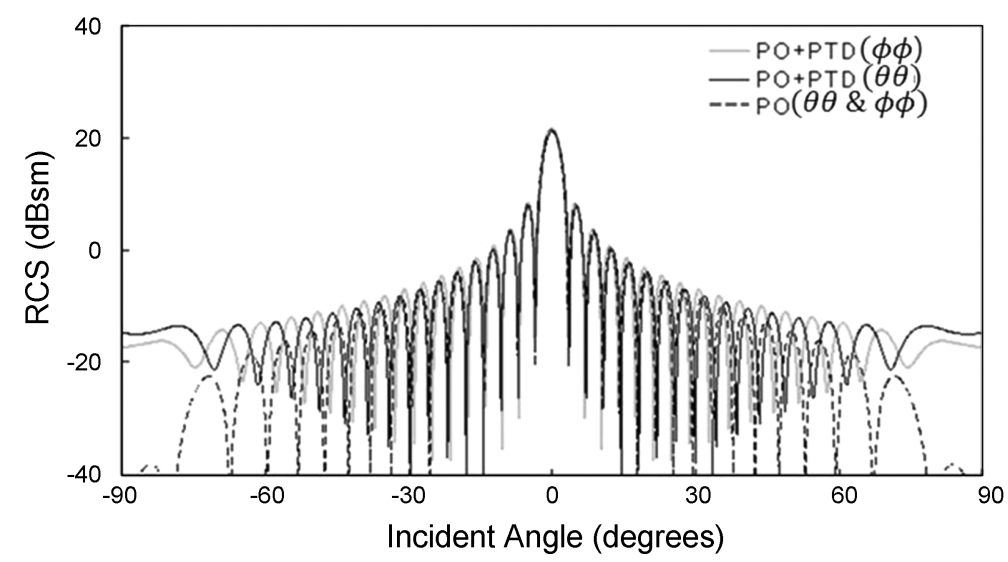

Fig. 13 RCS of rectangular plate considering edge diffraction.

\section{Closed-in weapon system}

To demonstrate the applicability of the presented system for electromagnetically large and complex targets, RCS analysis for a closed-in weapon system (CIWS) has been carried out only considering specular reflections. Fig. 14 shows the numerical model of the CIWS whose dimension is $4.7 \times 4.0 \times 3.7(\mathrm{~m})$ and the number of model elements is 4,270. The radar frequency is set to $18 \mathrm{GHz}$ in VV-polarizations. The range of azimuth angle varies from 0 to 360 degree in 1-degree step, and the elevation angle varies from 0 to 10 degree in 2-degree step. The maximum reflection order is set to 3. 
The RCS values for the elevation angle of 0 and 10 degree are representatively shown in Fig. 15 and the arithmetic mean RCS values with respect to the elevation angle are represented in Table 1. The results show good agreement compared with those calculated by SIGNET/RCS (BAE SYSTEMS, 2004).

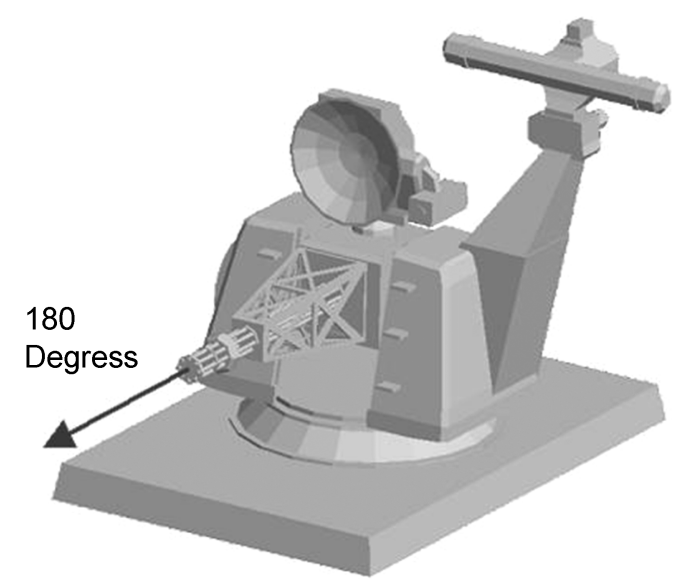

Fig. 14 Numerical model of CIWS.

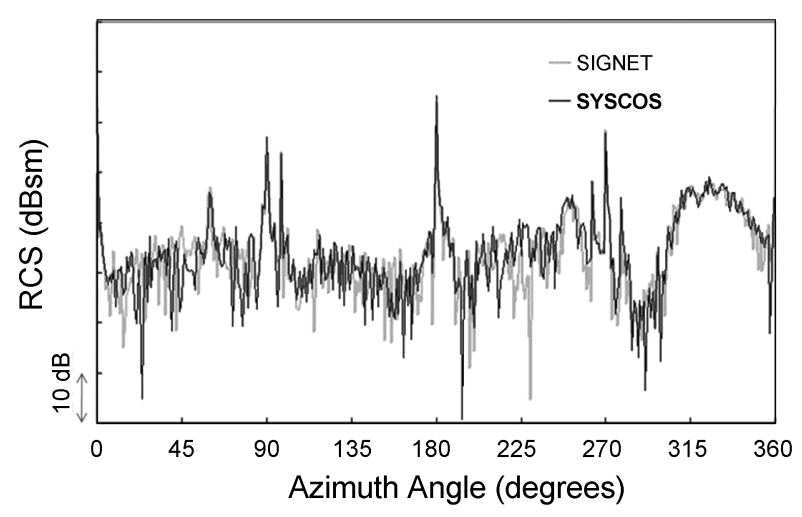

(a)

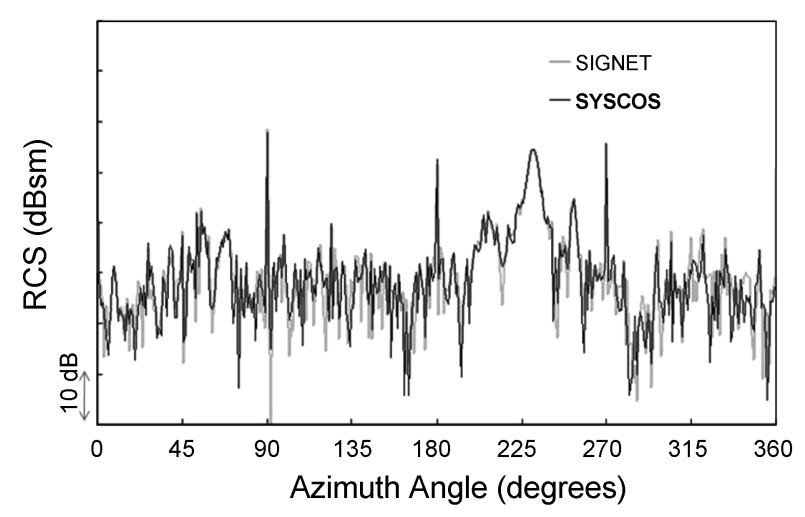

(b)

Fig. 15 RCS analysis result of CIWS compared to SIGNET/RCS, where the elevation angles are (a) 0 degree and (b) 10 degree.

Table 1 Mean RCS value of CIWS.

\begin{tabular}{|c|c|c|c|}
\hline \multirow{2}{*}{ Elevation Angle (degree) } & \multicolumn{3}{|c|}{ Mean RCS (dBsm) } \\
\cline { 2 - 4 } & SYSCOS & SIGNET & Difference \\
\hline 0 & 13.66 & 13.26 & 0.40 \\
\hline 2 & 8.64 & 8.60 & 0.04 \\
\hline 4 & 8.67 & 9.07 & -0.40 \\
\hline 6 & 8.34 & 8.31 & 0.03 \\
\hline 8 & 8.31 & 8.56 & -0.25 \\
\hline 10 & 8.93 & 9.75 & -0.82 \\
\hline
\end{tabular}

\section{Virtual naval ship}

To demonstrate the applicability of the presented system to RCS reduction design using radar absorbing material (RAM), the RCS analysis for a virtual naval ship has been carried only considering specular reflections. The model di-mension is 134.9 $\times 13.5 \times 29.5(\mathrm{~m})$ and the number of model elements is 31,350 . The radar frequencies are set to $4,8,10,12$ and $18 \mathrm{GHz}$ and the elevation angle is set to 4 degree. The other calculation conditions are same with those of CIWS. 
The RCS values of the ship model for the 5 radar frequencies are shown in Fig. 16. From the results, the several high peaks of RCS value are observed. Particularly, a high and narrow peak marked with "A" occurs around 24 degree of the azimuth angle due to multiple reflections between the side surfaces of the mast and the onboard equipment, whose scattering center analysis result in case of $12 \mathrm{GHz}$ radar frequency is shown in Fig. 17.

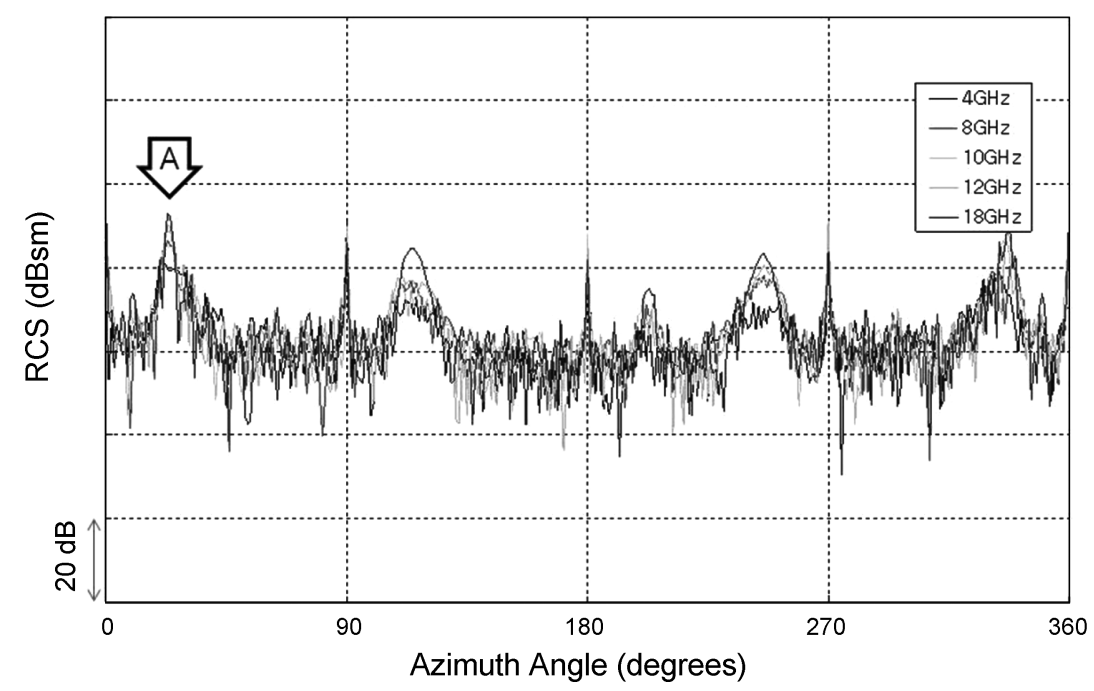

Fig. 16 Result of RCS analysis for the naval ship.

To reduce the peak, a multilayered RAM has been designed by a genetic algorithm method (Kim, et al., 2007b) and virtually attached on the corresponding surfaces. The material properties and the RCS reduction performance of the RAM are represented in Table 2 and Fig. 18, representatively.

The RCS levels of the ship before and after the RAM attachment are compared in Fig. 19. From the result, one can know that the RCS levels after the RAM attachment have been remarkably reduced around 24 degree of the azimuth angle.

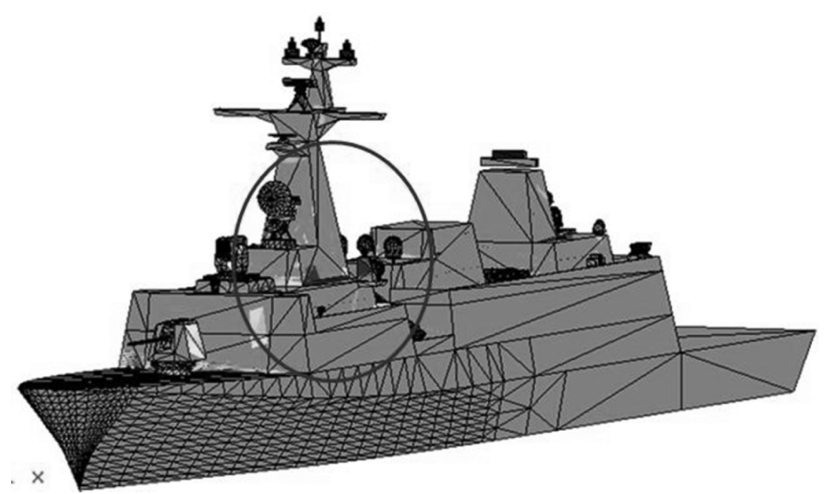

Fig. 17 Scattering center analysis result for 24 degree azimuth angle for the naval ship.

Table 2 Material properties of multilayered RAM.

\begin{tabular}{|c|c|c|c|}
\hline Layer \# & Thickness $(\mathrm{mm})$ & Relative Permittivity & Relative Permeability \\
\hline 1 & 0.2 & 15.0000 & $0.8108-4.6849 j$ \\
\hline 2 & 2.7 & $0.5886-1.9455 j$ & 1.0000 \\
\hline 3 & 1.4 & $0.5886-1.9455 j$ & 1.0000 \\
\hline 4 & 2.7 & $1.4468-0.7063 j$ & 1.0000 \\
\hline 5 & 2.4 & $0.5886-1.9455 j$ & 1.0000 \\
\hline Total & 9.4 & & - \\
\hline
\end{tabular}




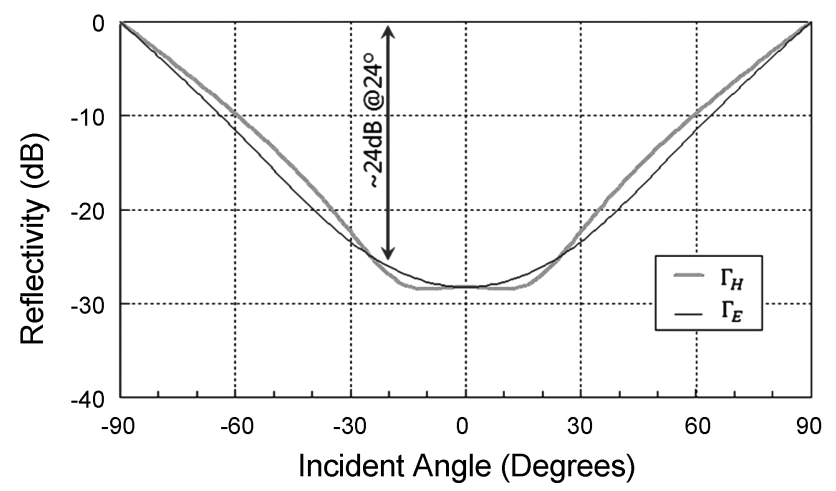

Fig. 18 RCS reduction performance (reflectivity) of multi-layered RAM with respect to incident angle.

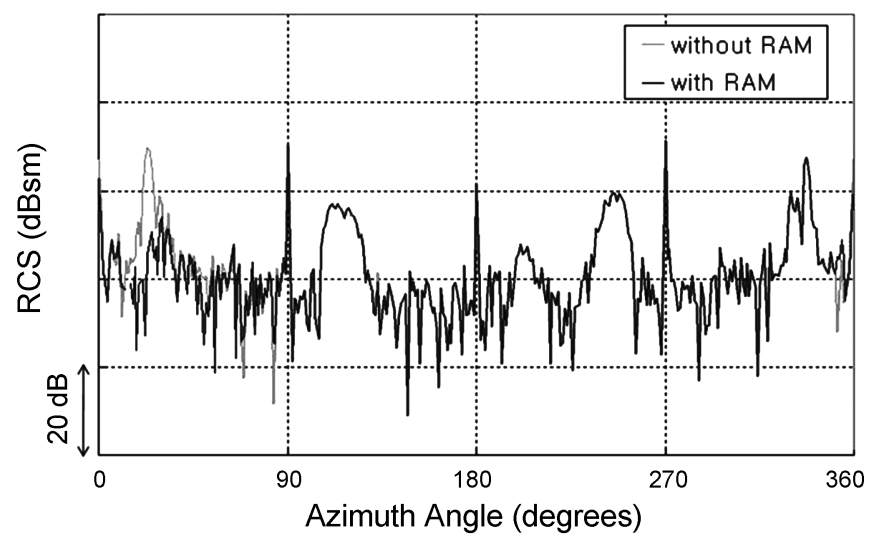

Fig. 19 RCS levels of the objective naval ship before and after RAM attachment.

\section{CONCLUSIONS}

A software system for complex object scattering analysis, named SYSCOS, has been developed by using the high frequency analysis methods of PO, GO, PTD, and a direct scattering center analysis method. The system has been composed of user-friendly functions to effectively carry out the RCS analysis and reduction design process of large and complex targets such as naval ships. Also, numerical validation tests for both various simple targets and real complex targets have been carried out and the results have proven the accuracy of the presented system and its applicability to real targets. Hence, it is expected that the presented system could be practically applied for the stealth design of real naval ships.

\section{ACKNOWLEDGEMENTS}

This work was supported by the National Research Foundation of Korea (NRF) grant funded by the Korea government (MEST) through GCRC-SOP (Grant No. 2011-0030669 and No. 2011-0030686).

\section{REFERENCES}

BAE SYSTEMS, 2004. Introduction to BAE SYSTEMS signature analysis tools. BAE SYSTEMS.

Biegel, G., Essen, H. and Brauns, R., 2004. Validation of RCS simulations with the code CADRCS for mm-wave fre-quencies. Radar 2004 - International Conference on Radar Systems.

IABG, 2002. RAMSES user manual. IABG, Ottobrunn.

Kim, K., Cho, D.S. and Kim, J.H., 2007a. High-frequency back-scattering cross section analysis of rotating targets. Journal of the Korea Institute of Military Science and Technology, 10(3), pp.16-24 (in Korean with English abstract).

Kim, K., Cho, D.S. and Kim, J.H., 2007b. Broad-band multi-layered radar absorbing material design for radar cross sec- 
tion reduction of complex targets consisting of multiple reflection structures. Journal of the Society of Naval Architects of Korea, 44(4), pp.445-450 (in Korean with English abstract).

Kim, K., Kim, J.H. and Cho, D.S., 2005a. RCS analysis of complex structures using object precision method. Journal of the Society of Naval Architects of Korea, 42(2), pp.159-164 (in Korean with English abstract).

Kim, K., Kim, J.H. and Cho, D.S., 2005b. A study on the effective scattering center analysis for radar cross section reduction of complex structures. Journal of the Society of Naval Architects of Korea, 42(4), pp.421-426 (in Korean with English abstract).

Kim, K., Kim, J.H. and Cho, D.S., 2009. Radar cross section analysis of marine targets using a combining method of physical optics/geometric optics and a Monte-Carlo simulation. Ocean Engineering, 36(11), pp.821-830.

Klement, D., Preissner, J. and Stein, V., 1988. Special problems in applying the physical optics method for backscatter computation of complicated objects. IEEE Transaction on Antennas and Propagation, 36(2), pp.228-237.

Knott, E.F., Tuley, M.T. and Shaeffer, J.F., 1993. Radar cross section. $2^{\text {nd }}$ Ed. Artech House Publisher.

Lee, S.W., Andersh, D.J., Reeves, D.D., Jeng, S.K. and Ling, H., 1993. User manual for XPATCH. DEMACO, Inc.

Lee, S.W., Ling, H. and Chou, R., 1988. Ray-tube integration in shooting and bouncing ray method. Microwave and Optical Technology Letters, 1(8), pp.286-289.

Simpson, S.H., Galloway, P. and Harman, M., 1998. Applications of EPSILON ${ }^{\mathrm{TM}}$ : a radar signature prediction and analysis tool. International Radar Symposium '98, Germany, 1998.

Suk, S.H., Seo, T.I., Park, H.S. and Kim, H.T., 2001. Multi-resolution grid algorithm in the SBR and its application to the RCS calculation. Microwave and Optical Technology Letters, 29(6), pp.394-397. 